\title{
EPIDEMIOLOGICAL PROFILE OF PREGNANT WOMEN WITH SYPHILIS IN THE CITY OF MACAÉ, RIO DE JANEIRO
}

\author{
Adail Orrith Liborio Neto ${ }^{1}$, Juliane de Freitas Santos ${ }^{2}$, \\ Carlos Alexandre Ribeiro Goulart ${ }^{3}$
}

Clin Biomed Res. 2020;40(4):206-212
1 Faculdade de Medicina, Universidade
Federal do Rio de Janeiro. Macaé,
RJ, Brasil.

2 Faculdade de Medicina, Universidade Federal do Rio de Janeiro. Macaé, RJ, Brasil.

3 Faculdade de Medicina, Universidade Federal do Rio de Janeiro. Macaé, RJ, Brasil.

Corresponding author: Adail Orrith Liborio Neto adail_orrith@hotmail.com Faculdade de Medicina, Universidade Federal do Rio de Janeiro Rua Aloízio da Silva Gomes, 50 27930-560, Macaé, RJ, Brasil.

\section{ABSTRACT}

Introduction: Gestational and congenital syphilis are diseases that can be prevented. Practices routinely carried out in prenatal care are effective for the prevention of cases. The prevalence of syphilis in pregnant women is considered an indicator of prenatal care quality. The objective is to define the epidemiological profile of pregnant women diagnosed with syphilis in the city of Macaé - Rio de Janeiro from 2010 to 2018.

Methods: A cross-sectional retrospective study covering from 2010 to 2018 was conducted to assess the general prevalence in the period and annual incidence rates in records and database from the Care Center of Women and Children and from the Macaé Public Hospital.

Results: A total of 535 cases of syphilis were reported in pregnant women. The highest number of reported cases was observed in $2018(n=151)$, with a detection rate of $38.4 \%$ per 1000 live births, meaning an increase of $37 \%$ compared to 2010 . Most cases were diagnosed in the third trimester of pregnancy ( $n=194$ cases). The majority of women were aged $20-29$ years $(n=322)$ and were housewives $(n=248)$. The predominant skin color was brown, accounting for $32.4 \%(n=180)$ of the cases, followed by black skin $(16.9 \% ; n=124)$. The majority of patients $(n=124)$ had incomplete elementary school education. The annual incidence rate of congenital syphilis increased from 1.4 to 17.2 cases per 1,000 live births between 2010 and 2018 .

Conclusions: There was an increased prevalence of syphilis in the city of Macaé, especially after 2015, with a peak of cases in 2018.

Keywords: Syphilis; pregnancy; women's health services; women's health, pregnant women

\section{INTRODUCTION}

Syphilis is an infectious, systemic, worldwide disease with chronic clinical course, caused by the bacterium Treponema pallidum, which has humans as the single host, transmitter and reservoir ${ }^{1}$.

In Brazil, the number of reported cases of syphilis in pregnant women has increased every year. Between 2008 and 2018, particularly from 2010 onwards, there was a progressive increase in the incidence of gestational syphilis and hence congenital syphilis. In 2006, there was a rate of 2.0 cases per thousand live births; in 2016, 37,436 cases of syphilis were reported in pregnant women and 20,474 cases of congenital syphilis in Brazil, yielding a rate of 6.8 cases per thousand live births ${ }^{2}$.

The rate of vertical transmission of syphilis in untreated women is $50-85 \%$ in the primary and secondary stages of the disease. This rate is reduced to $30 \%$ in latent and tertiary stages. Syphilis has great impact on public health due to the high frequency with which it produces severe outcomes for pregnant women and for children, such as premature birth, fetal and neonatal death, and congenital infection of the newborn ${ }^{3}$.

In addition to representing a major public health problem, control of syphilis is still a challenge for many countries. In 2008, the World Health 
Organization (WHO) estimated that 12 million people, including about two million pregnant women, were infected with Treponema pallidum ${ }^{4}$, making congenital syphilis more common than perinatal HIV infection ${ }^{5}$. Overall, the Americas region has the highest incidence rate of syphilis and accounts for up to $25 \%$ of the 2 million annual cases of gestational syphilis. The prevalence of gestational syphilis in the Americas (Canada, Latin America and the Caribbean, and the United States) varies by country from $0.01 \%$ to $7.0 \%{ }^{6}$.

Gestational and congenital syphilis are diseases that can be prevented. Practices routinely carried out in prenatal care are effective in preventing cases. These are called sentinel events, since the disease can be prevented by effective health actions that require retrospective investigation of cases for information on the assistance and that propose relevant measures.

The prevalence of syphilis in pregnant women is considered an indicator of prenatal care quality. Therefore, the increase in its prevalence over the years underscores the need for actions directed to the control of syphilis in primary care and bring knowledge to society that the increased rates of this disease has a negative impact on the lives of pregnant women and also of their children.

The objective of the present study was to define the epidemiological profile of pregnant women diagnosed with syphilis in the city of Macaé, Brazil, between 2010 and 2018, to assess the risk factors for the transmission of this disease, and to check the provision of prenatal care, treatment of pregnant women diagnosed with syphilis, and concurrent patient-partner treatment.

\section{METHODS}

A cross-sectional retrospective study covering from 2010 to 2018 was conducted to assess the general prevalence in the period and annual incidence rates. The project was approved by the Ethics Committee for Research involving human beings (Comitê de Ética em Pesquisa, CEP) of Universidade Federal do Rio de Janeiro, Campus Macaé, under Certificate Presentation Ethics for Assessment (Certificado de Apresentação de Apreciação Ética, CAAE) reference number 01896018.2.0000.5699. This study consisted of consultations with data from the Notifiable Diseases Information System (Sistema de Informação de Agravos de Notificação, SINAN), as well as of documentary research in records and database from the Care Center of Women and Children and the Macaé Public Hospital.

\section{RESULTS}

In the period 2010-2018, 535 cases of syphilis in pregnant women and 206 cases of congenital syphilis were reported in the city of Macaé. The year 2018 had the highest number of reported cases of gestational syphilis $(n=151)$, with a detection rate of $38.4 \%$ per 1,000 live births (Chart 1), meaning an increase of $0.2 \%$ over the previous year, of $37 \%$ compared to 2010 , and of $28.02 \%$ compared to the average rate for the period analyzed in this study.

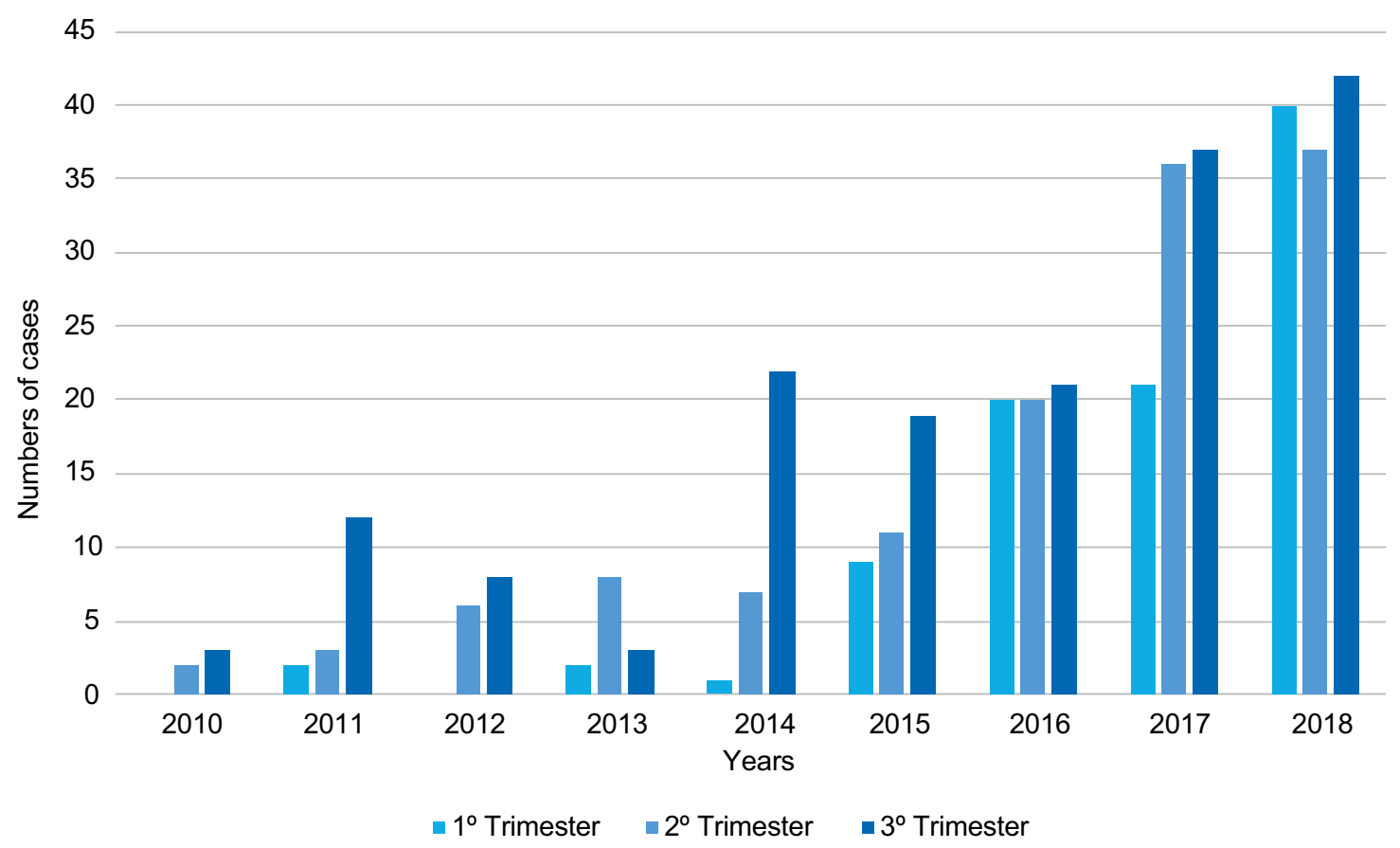

Chart 1: Cases of syphilis in pregnant women according to gestational age and year of notification in Macaé. 
Figure 1 shows the map of the city of Macaé with markings of the spatial distribution of gestational syphilis in the city from the neighborhoods that have Family Health Strategy (FHS) units. In a descending order of number of cases, the majority of cases were observed in the following districts: Nova Holanda, Lagomar, Parque Aeroporto, Fronteira, Barra de Macaé, Malvinas, Ajuda, Botafogo, and Aroeira.

An analysis of the number of cases of pregnant women with syphilis according to gestational age revealed that the majority of cases were diagnosed in the third trimester of pregnancy ( $n=194$ cases), ranging from 3 cases in 2010 up to 42 cases in 2018 (Chart 1). Most patients with syphilis $(n=322)$ were aged from 20 to 29 years old (average 25 years, range $13-40$ years), with a representative percentage of $49 \%$ (Table 1). With regard to occupation, most patients affected by the disease were housewives $(n=248)$, followed by students $(n=88)$, and finally by unemployed $(n=23)$ (Chart 2$)$.

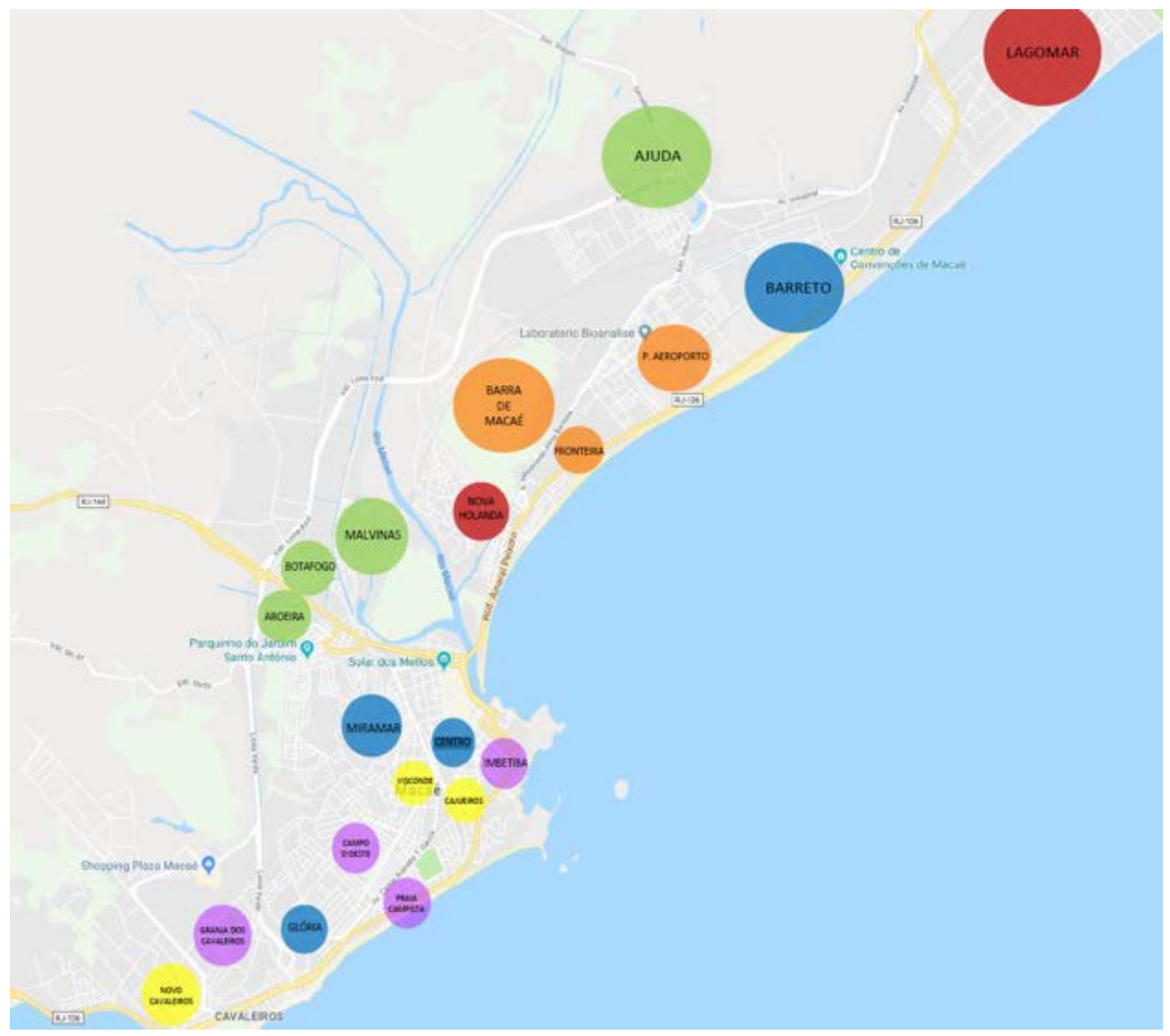

Figure 1: Map of the city of Macaé. Markings demonstrate districts with Family Health Strategy units. 
Table 1: Distribution of cases according to age and concurrent patient-partner treatment.

\begin{tabular}{lc}
\hline Age & $\%$ \\
\hline $10-14$ years & $2 \%$ \\
$15-19$ years & $38 \%$ \\
$20-29$ years & $49 \%$ \\
$30-39$ years & $10 \%$ \\
$40-49$ years & $1 \%$ \\
\hline Concurrent patient partner treatment & $\%$ \\
\hline Yes & $13 \%$ \\
No & $45 \%$ \\
Unknown & $42 \%$ \\
\hline
\end{tabular}

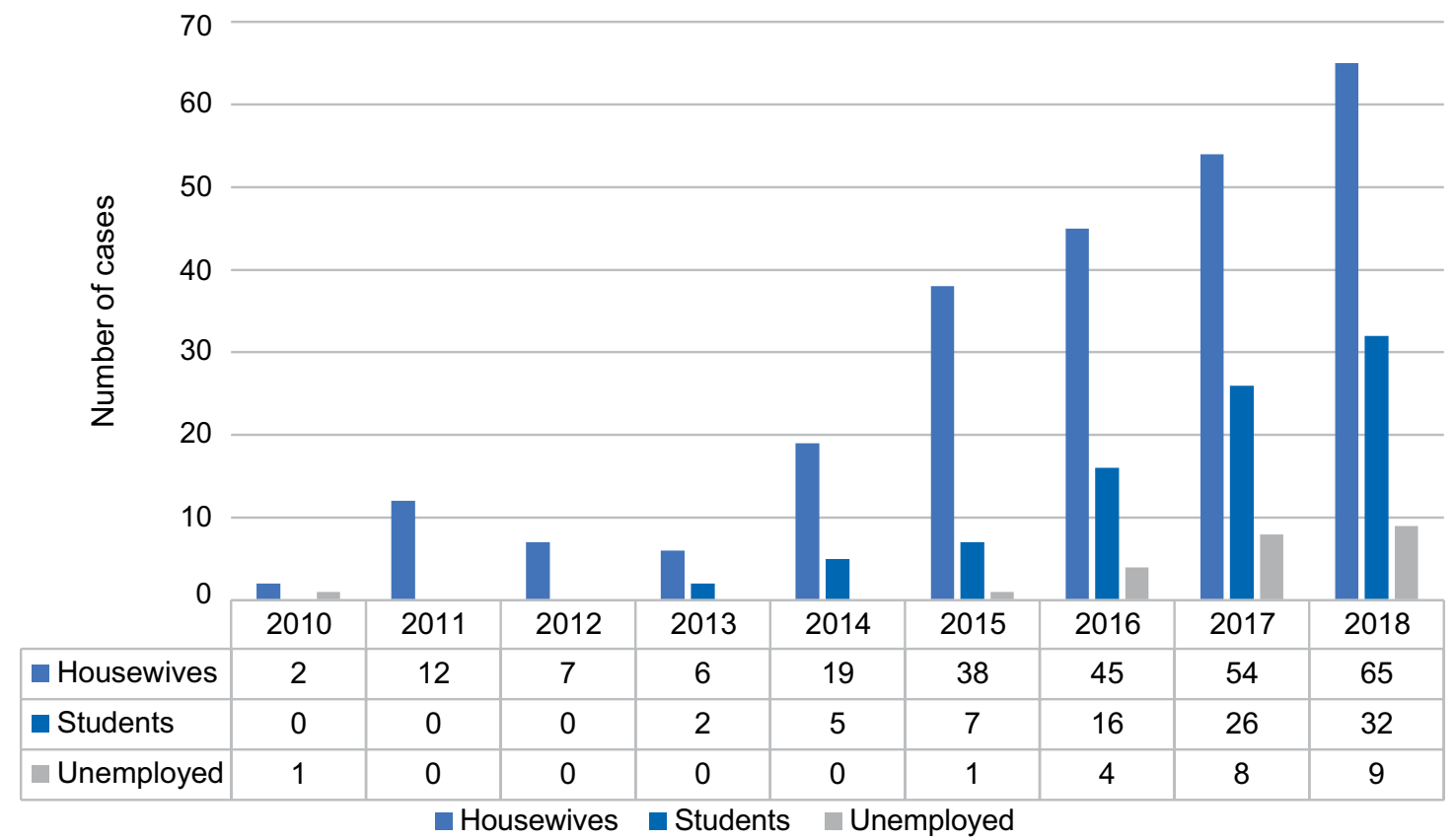

Chart 2: Number of cases of syphilis in pregnant women according to occupation.

It was found that $32.4 \%(n=180)$ of patients were of mixed race, of whom the highest percentage $(61.5 \%)$ was observed in 2013 , followed by $16.9 \%(n=124)$ of patients who were black. However, race was unknown for $28.3 \%(n=217)$ of cases. The great majority of women $(n=124)$ had incomplete elementary school education; of these, $75 \%(n=93)$ studied up to the $5^{\text {th }}$ to the $8^{\text {th }}$ grade.

As a result of these data, it was observed that the annual incidence rate of congenital syphilis in Macaé increased from 1.4 to 17.2 cases per 1,000 live births between 2010 and 2018 (Chart 3).
Of all cases of congenital syphilis, $85.9 \%$ of the mothers had prenatal care during pregnancy, and $47.7 \%$ were diagnosed prenatally. Furthermore, $45 \%$ of partners of mothers who underwent prenatal care received concurrent treatment, but this information was missing in $42 \%$ of records (Table 1 ). Data on the prescribed treatment plan for gestational syphilis are available only 2015 onwards. A total of 314 pregnant women were treated with penicillin $(69.5 \%)$, six with another type of antibiotic $(1.6 \%)$, and there were 108 cases $(23.1 \%)$ of missing information regarding treatment. 


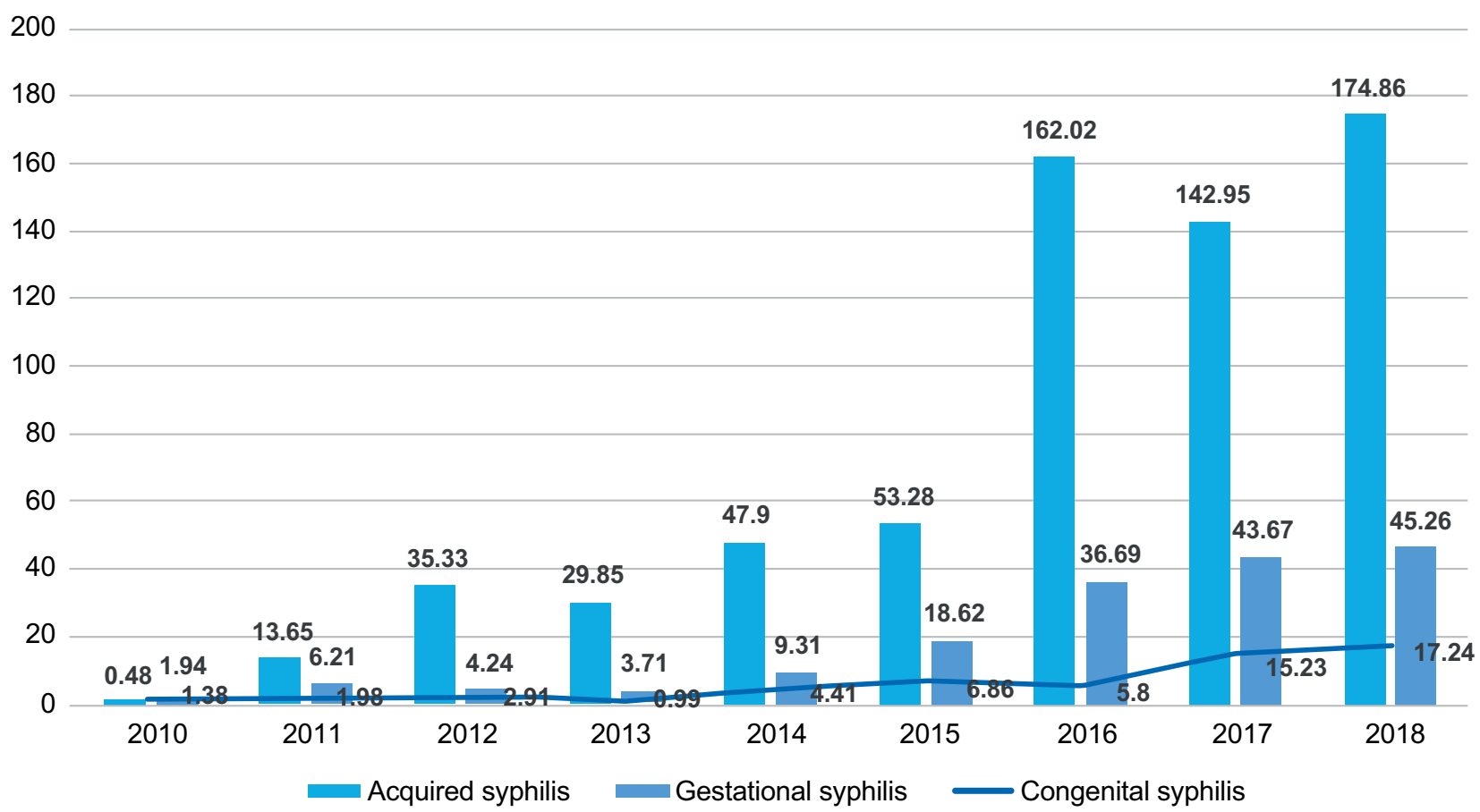

Chart 3: Incidence rates of acquired syphilis, gestational syphilis, and congenital syphilis per 1,000 live births.

\section{DISCUSSION}

There was an increase in the incidence of women with syphilis and consequently an increased incidence of congenital syphilis in Macaé (Chart 3). This increase has also been reported in other cities in Brazil and worldwide ${ }^{2,5}$. With the increased coverage of primary care conducted by FHS units in Brazil and of primary health care practices such as prenatal care and child care, a better control of gestational syphilis and of vertical transmission was expected.

The results of this study show that the incidence of syphilis is higher in the third trimester of pregnancy. By correlating this data with the medical practice during medical internship performed in hospitals in the city of Macaé, we observe that this finding can be explained by late diagnosis of the disease, which often occurs only during tests for admission to the maternity unit, which is the end of the care line. This is demonstrated by the fact that the Municipal Public Hospital has notified approximately two-thirds of all cases reported in the city for years, showing that prenatal care is often performed inadequately.
Another possibility to explain the incidence of diagnosis in the third trimester of pregnancy would be the overcrowding of primary health care services, which would cause a delay in scheduling consultations and prenatal examinations, in addition to a delay in the release of results of complementary tests.

An epidemiological finding that reveals the dark side of the disease in modern society was the large number of housewives infected by syphilis (Chart 2). The authors think of two possibilities to explain these numbers: the first one considers that these women do not own and fixed income, due to their financial and psychological dependence on their partner, and thus could be more vulnerable to his desire not to use condoms during sexual intercourse, making these women more vulnerable to the disease, reinforcing the sexist nature of today's society. The second possibility considered was that couples with steady relationships usually decide to quit using condoms because of the privilege of being in a stable relationship, believing that their partner is monogamous. The main cause of infection and re-infection is known to be inadequate treatment of sexual partner ${ }^{7}$, an analysis that 
can also be confirmed by the data in Table 1 . This table point to the large number of untreated partners or with unknown treatment status the notification forms, highlighting the importance of recycling the health team regarding the incentive for partner treatment, use of condom, and proper completion of the notification form. Partner's adherence to the treatment of syphilis is an important item in the control of disease incidence, because although pregnant women receive the three doses of medication, the treatment is considered incomplete without their partner's adherence, leaving the mother and the fetus still under vulnerability.

The special distribution of cases of syphilis shown in Figure 1 reveals that the highest disease incidence is found in the most populated areas. FHS units are present in most areas of Macaé, and this study observed that the districts with the highest number of cases of gestational syphilis are Nova Holanda, Lagomar, Parque Aeroporto, Fronteira, Barra de Macaé, Malvinas, Ajuda, Botafogo, and Aroeira. By analyzing the map, it is noticed that these districts are located in the northern outskirts of the city, and most of them are located above Rio Macaé. These districts had an exponential disorganized population growth. Individuals living in districts located in the south of the city show a smaller number of cases, precisely because they have high purchasing power, easy access to health services. Moreover, these districts exhibit a slower and more organized population growth and are nearby tourist sites; moreover, and real estate located in these districts has a higher price. These variables reduce the incidence of syphilis in pregnant women, but are not a full protective factor, because there were cases of pregnant women with syphilis in the most privileged districts of the city, which shows that people cannot be stigmatized only due to the presence or absence of social and economic vulnerabilities.

Regarding age, the highest incidence was found among pregnant women aged 20-29 years, with a percentage representing $49 \%$, followed by those aged 15-19 years. Both age groups include sexually active populations, and these findings confirm data from other studies ${ }^{7,8}$. As for skin color, brown skin was the predominant one, with 2013 being the year with the highest percentage of cases with this skin color $(61.5 \%)$, followed by black skin. However, it is important to mention that skin color was reported as unknown in $28.29 \%$ of the cases. The high predominance of mixed race results from the fact that Macaé is a coastal city with an economy based on oil exploration and that attracts people from all regions of Brazil and the world. This justifies the mixed racial profile of the population and the predominance of brown skin color.

The vast majority of pregnant women had not completed elementary school education, most of which studied up to the $5^{\text {th }}$ to the $8^{\text {th }}$ grade, followed by complete primary education and incomplete higher education. Features such as skin color, low level of education, women at reproductive age, and lack of paid occupation were statistically associated with gestational syphilis, which is also shown in other studies ${ }^{4,7,8}$. However, it is not possible to generalize the cases of syphilis to a type of exclusively of the poorest population profile. On the contrary, regardless of social or financial condition, any individual can acquire the infection, but the risk is statistically higher in vulnerable populations ${ }^{8}$.

The results showed the need to invest in the screening of pregnant women susceptible to infection with $T$. pallidum and explained the low level of information about the disease provided in municipal health units that perform prenatal care. The multidisciplinary team working at the FHS units is responsible to make active search of pregnant women missing prenatal consultations, to take action to raise awareness of the population assistedby the FHS units on the risks of unprotected sexual practice and on the importance of the full treatment of the couple, especially among the most vulnerable population.

There was an increased incidence of syphilis in the city of Macaé, especially after 2015, with a peak of cases in 2018. The factors that contributed to this increase were sexually active population, incomplete education, pregnant women's occupation, and lack of partner's adherence to treatment. Despite the high prenatal coverage, there is still a difficulty in implementing actions of primary care for the prevention and management of gestational syphilis. We believe that to these numbers can be reduced by performing early educational interventions in the care line. It is essential that health professionals and the community raise awareness about the importance of early diagnosis and effective treatment of women and their partners, contributing to the protection of the mother-fetus binomial. 


\section{REFERENCES}

1. Centers for Disease Control and Prevention. Sexually transmitted diseases treatment guidelines, 2015. MMWR MMWR Morb Mortal Wkly Rep. 2015;64(3):1-138.

2. Brasil. Ministério da Saúde. Secretaria de Vigilância em Saúde. Departamento de Vigilância, Prevenção e Controle das IST, do HIVIAids e das Hepatites Virais. Boletim epidemiológico: síflis. Brasilia, DF; 2017 [cited 2021 Feb 20]. Available from: http://www.aids.gov.br/ pt-br/pub/2017/boletim-epidemiologicode-sifilis-2017

3. Brasil. Ministério da Saúde. Secretaria de Vigilância em Saúde. Programa Nacional de DST e Aids.. Diretrizes para o controle da síflis congênita. Brasilia, DF: Ministério da Saúde; 2006 [cited 2021 Feb 20]. Available at: http://bvsms.saude. gov.br/bvs/publicacoes/diretrizes_ controle_sifilis_congenita.pdf

4. Araújo $\mathrm{CL}$, Shimizu HE, Sousa AIA, Hamann EM. Incidence of congenital syphilis in Brazil and its relation to the Family Health Strategy. Rev Saude Publica. 2012;46(3):479-86.

5. Pan American Health Organization. 2010 Situation analysis: elimination of mother-to-child transmission of HIV and congenital syphilis in the Americas. Washington, DC: PAHO; 2011 [cited $2021 \mathrm{Feb}$ 20]. Available from: https://www. paho.org/hq/dmdocuments/2012/ Elimination-print.pdf
6. Gomez GB, Kamb ML, Newman LM, Mark J, Broutet N, Hawkes SJ. Untreated maternal syphilis and adverse outcomes of pregnancy: a systematic review and metaanalysis. Bull World Health Organ. 2013;91(3):217-26.

7. Cavalcante PAM, Pereira RBL, Castro JGD. Syphilis in pregnancy and congenital syphilis in Palmas, Tocantins State, Brazil, 2007-2014. Epidemiol Serv Saude. 2017;26(2):255-64.

8. Padovani C, Rossetto RO, Pelloso SM. Syphilis in during pregnancy: association of maternal and perinatal characteristics in a region of southern Brazil. Rev Lat Am Enfermagem. 2018;26:e3019.

Received: Mar 14, 2020 Accepted: Nov 29, 2020 